\title{
Diversity and Floristic Composition in the Analogous Vegetation of Indiviso, Baquerizo Moreno, Tungurahua
}

\section{Diversidad y Composición Florística en el Vegetacion Análoga de Indiviso, Baquerizo Moreno, Tungurahua}

\author{
J. Caranqui ${ }^{1}$ and M. Ortíz ${ }^{2}$ \\ ${ }^{1}$ Herbario Escuela Superior Politécnica del Chimborazo CHEP, Riobamba, Ecuador \\ ${ }^{2}$ G.A.D.PR. Baquerizo Moreno, Galápagos, Ecuador
}

Memories II International

Congress Forests and

Agroforestry for the 21st

Century

Corresponding Author:

J. Caranqui

jcaranqui@yahoo.com

Published: 21 January 2021

Production and Hosting by

Knowledge E

(c) J. Caranqui and M.

Ortíz. This article is

distributed under the terms of the Creative Commons

Attribution License, which permits unrestricted use and redistribution provided that the original author and source are credited.

\section{Abstract}

This research study contributes to the knowledge of the diversity and similarity of vegetation more than $5 \mathrm{~cm}$ diameter at breast height (DBH); the study was carried out in the montane forest located in Tungurahua, Pillaro, Baquerizo Moreno, Indiviso (01'18'S, $78^{\circ} 30^{\prime} \mathrm{W}$ ), at an altitude of $30,400 \mathrm{~m}$. A transect of $1000 \mathrm{~m}^{2}$ was made, divided into 5 plots of $50 \times 4 \mathrm{~m}$. The species were identified by the ESPOCH Herbarium, and the values calculated were relative density (DR) and relative dominance (DMR) to obtain the Importance Index (IV). 18 families, 29 genera, and 33 species were found, corresponding to 249 individuals, in which the largest number of individuals were accounted for by Escallonia myrtilloides (8.03\%), Macleania rupestris (7.63\%), Vaccinium floribundum (6.02\%), Gynoxis buxifolia and Miconia bracteolata with $5.62 \%$, Bacharis teindalensis and Blechnum loxensis with $4.82 \%$, and Maytenus vertillata, Hypericum laricifolium, Tibouchina lepidota, Brachyotum ledifolium, Sympoccos with 4.02\%; the remaining species had lower values. The reason for finding both species of shrub paramo and high montane forest is not so clear, since physiognomically the area under study was a high montane forest. Furthermore, no studies have been conducted in other transition zones, but for this reason, a high number of species of two types of vegetation is found.

Keywords: analogue flowering, shrub paramo, high montane forest, indiviso.

\section{Resumen}

El presente trabajo contribuye al conocimiento de la diversidad y similitud de la vegetación mayor de $5 \mathrm{~cm}$ de diámetro a la altura del pecho (DAP); el estudio se realizó en el bosque montano localizados en provincia de Tungurahua, cantón Pillaro, parroquia Baquerizo Moreno, sector Indiviso, en las ccordenadas 01^18'S; 78³0'W, altitud 30.400 m.s.n.m. Se realizó un transecto de $1000 \mathrm{~m}^{2}$, divididos en 5 subtransectos de $50 \times 4 \mathrm{~m}$. Las especies se identificaron el Herbario ESPOCH, los datos calculados fueron Densidad relativa (DR), dominancia relativa (DMR) para obtener Indice de Importancia (IV). se encontraron 18 familias, 29 géneros, 33 especies que corresponden a 249 individuos, en las cuales el mayor número de individuos cuenta Escallonia myrtilloides (8,03\%), Macleania rupestris (7,63\%), Vaccinium floribundum (6,02\%), Gynoxis buxifolia y Miconia bracteolata con 5,62\%, Bacharis teindalensis y Blechnum loxensis con 4,82\%, Maytenus vertillata, Hypericum laricifolium, Tibouchina lepidota, Brachyotum ledifolium, Sympoccos quítense con 4,02\%; el resto con 
valores inferiores. La explicación de tanto especies de páramo arbustivo y bosque montano alto no están tan claros, ya que fisionomicamente fue un bosque montano alto esta zona en estudio. Además no se han encontrado estudios en otras zonas de transición, pero por ello se encuentra un número alto de especies de dos tipos de vegetación.

Palabras Clave: vegetación análoga, páramo arbustivo, bosque montano, indiviso.

\section{Introducción}

\subsection{Bosques montanos}

Los bosques de montaña se caracterizan por una enorme diversidad biológica (tan diversa quizás como la famosa selva tropical lluviosa), pero también por regular los importantes caudales hídricos de los ríos que atraviesan el continente y por sobre todo, por compartir una historia de uso y de oferta de recursos en forma interrumpida con la humanidad, durante por lo menos la última decena de miles de años [1]. Sin embargo, hoy se presentan como uno de los sistemas más frágiles a la intervención humana y sobre el cual está cayendo con inusual fuerza los procesos de degradación por sobre-utilización y conversión en sistemas agrícolas y campos de pastoreo [2].

El bosque montano, un tipo de vegetación donde se esperan altos niveles de diversidad, debido a las barreras biogeográficas que son tan frecuentes en las montañas. La diversidad y composición florística son los atributos más importantes para diferenciar o caracterizar cada complejo y/o comunidad vegetal [3]. Por otro lado, la composición florística de un bosque es determinada por el conjunto de especies de plantas que lo componen y es tradición medirla considerando la frecuencia, abundancia o dominancia de las especies [4].

Según Sierra [5], el bosque siempreverde montano alto, se extiende dentro de una franja más amplia que en las estribaciones orientales, desde los 2900 hasta los 3600 m.s.n.m. Incluye la 'Ceja Andina' o vegetación de transición entre los bosques montanos altos y el páramo (por ejemplo alrededor de la laguna de Papallacta). El bosque húmedo montano es similar al bosque nublado en cuanto a su fisonomía y la cantidad de musgos y plantas epífitas. El suelo tiende a estar cubierto por una densa capa de musgo y los árboles tienden a crecer irregularmente, siendo ésta la fisonomía típica de los bosques altos andinos, con troncos ramificados desde la base y en algunos casos muy inclinados o casi horizontales.

\subsection{Páramo arbustivo}

Los ecosistemas de páramo son categorizados como uno de los sitios con mayor biodiversidad del mundo, considerado por algunos como archipiélago biológico $[6,7]$. En el Ecuador la franja altitudinal entre los 3000 hasta los 4500 m.s.n.m. contiene casi el $30 \%$ de las especies de plantas vasculares [8]; esto demuestra la gran representatividad 
que tiene este ecosistema y la importancia de conocer cuál es su diversidad florística, ya que esta clase de información servirá como punto de partida para el monitoreo del cambio climático y el comportamiento de la flora.

Según [9] el páramo en realidad posee una variedad mucho mayor de lo que la imagen clásica ('lugar yermo desprovisto de árboles') nos haría pensar. Los páramos, en el Neotrópico, cubren alrededor del $2 \%$ de la superficie de los paises de esa región; tiene cerca de 125 familias, 500 géneros y 3400 especies de plantas vasculares. En términos del Ecuador, aún no se conoce el número exacto de especies de plantas que viven en los páramos, pero [10] sugiere que son alrededor de 1500 especies. También [11] manifiesta que hasta la fecha, se han registrado para los páramos del Ecuador un total de 1524 especies, siendo para este ecosistema y en relación a su tamaño, el país con la flora más diversa de la región andina. De las especies registradas para Ecuador, se estima que alrededor de 628 especies de plantas son endémicas, esto equivale al $15 \%$ de toda la flora endémica del país y el $4 \%$ del total de su flora [12]. De acuerdo a [13] el 75\% de éstas especies endémicas están amenazadas y apenas el $48 \%$ están dentro de áreas protegidas.

Se encuentra sobre los 3100 m.s.n.m. Las hierba en penacho son reemplazadas por arbustos, hirbas de varios tipos, plantas en roseta $y$, especialmente en los páramos más húmedos, por plantas de almohadilla. Pequeños árboles de los géneros Polylepis y Escallonia puden ocurrir. Flora característica. Azorella pedunculata, A. aretioides, A. corymbosa (Apiaceae); Baccharis spp., Culcitium spp., Chuquiraga juseeieu, Diplostephium rupestre, Loricaria sp.; Oritrophium spp., Senecio spp., Werneria humilis, W. nubigena (Asteraceae); Draba aretioides, Draba sp. (Brassicaceae); Siphocampylos asplundi (Campanulaceae); Gentiana spp., Halenia spp. (Gentianaceae); Isolepis spp. (Isoetaceae); Distichia tolimensis (Juncaceae); Lupinus alopecuroides (Fabaceae); Lycopodium spp. (Lycopodiaceae); Plantago rigida (Plantaginaceae); Lachemilla orbiculata (Rosaceae); Valeriana spp. (Valerianaceae). Las plantas de almohadillas pertenecen a muchas familias diferentes como Asteraceae, Apiaceae, Ericaceae, Geraniaceae, Plantaginaceae, Brassicaceae y Juncacea [5].

Por lo manifestado anteriormente, el objetivo de este trabajo es contribuir al conocimiento de la diversidad y similitud de la vegetación mayor de $5 \mathrm{~cm}$ de diámetro a la altura del pecho (DAP), encontrada en el bosque montano análogo (indiviso) de Baquerizo Moreno, Tungurahua.

\section{Materiales y Métodos}

\section{1. Área de estudio}

Este estudio se realizó en el bosque montano según [5]. Localizados en provincia de Tungurahua, cantón Pillaro, parroquia Baquerizo Moreno, sector Indiviso, en las ccordenadas $01^{\circ} 18^{\prime}$ S; $78^{\circ} 30^{\prime} \mathrm{W}$, altitud 30.400 m.s.n.m.: 


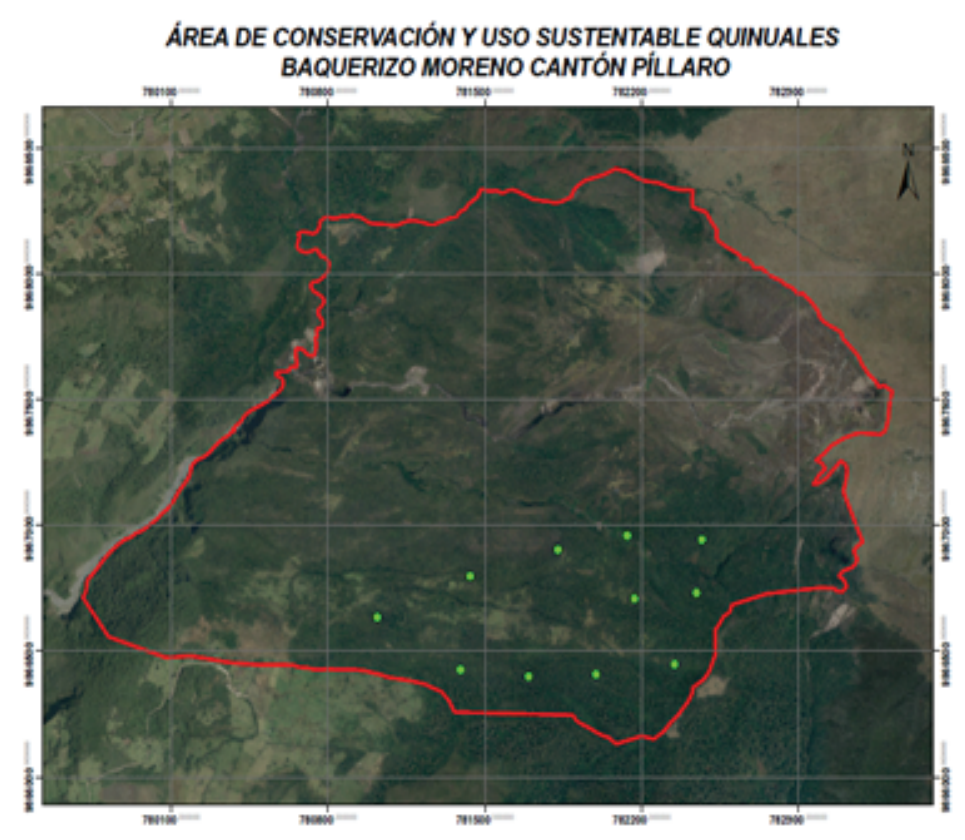

\section{Figure 1}

Localización del área de estudio.

\subsection{Toma de datos}

En el sitio se instaló una área muestreal de 0.1 hectárea, divididos en 2 transectos de $50 \times 4 \mathrm{~m}$ que es variante de los transectos realizados de Gentry, adaptados en: [14-17], ya que estos eran de 10 juegos de transectos de $50 \times 2 \mathrm{~m}$. Utilizando transectos amplios reduce los efectos de micro hábitats en los resultados generales, y tomando en cuenta la fragmentación del bosque que en la actualidad ocurre, los transectos de $50 \times 4 \mathrm{~m}$ nos ayudan a estandarizar el área de muestreo. Los siete transectos son del mismo tamaño lo que permite una comparación entre ellos.

El trabajo de campo se realizó el 13 de diciembre del 2018. Se tomó el DAP (diámetro a la altura del pecho) de las especies mayores de $5 \mathrm{~cm}$. Se colectaron especímenes botánicos de la mayoría de los individuos marcados (incluyendo todas las especies no definitivamente identificados en el campo) un duplicado de muestras. Las muestras se identificará en el Herbario de la Escuela Superior Politécnica de Chimborazo (CHEP). Para mayor información de las especies encontradas se revisó el Catálogo de Plantas Vasculares [18], y la actualización se consultó en la base de datos Trópicos (www. tropicos.org) del Missouri Botanical Garden [19].

\subsection{Análisis de datos}

Se realizaron los siguientes cálculos [17]:

$$
A B=\pi(D)^{2} / 4
$$


donde: AB: Área basal; D: DAP (Diámetro a la altura del pecho [1,30 m.]); П: 3.1416 (constante); Densidad: Número de árboles en la parcela; Densidad Relativa (DR): Número de árboles de una especie/número de árboles en la parcela*100; Dominancia Relativa (DMR): Área basal de una especie/Área basal total de todos los árboles en la parcela*100; Índice de Valor (IV): DR + DMR.

Además se generó un listado general de especies con sus respectivas frecuencias en cada uno de los transectos, con los cuales se obtuvo: Riqueza, diversidad (índice de Simpson), índice de similitud [20], calculados en el programa PAST.

\section{Resultados}

En el cuadro 1 se encuentra 18 familias, 29 géneros, 33 especies que corresponden a 249 individuos, en las cuales el mayor número de individuos cuenta Escallonia myrtilloides (8,03), Macleania rupestris (7,63), Vaccinium floribundum $(6,02)$, Gynoxis buxifolia y Miconia bracteolata con 5,62, Bacharis teindalensis y Blechnum loxensis con 4.82, Maytenus vertillata, Hypericum laricifolium, Tibouchina lepidota, Brachyotum ledifolium, resto Sympoccos quítense con 4,02; el con valores inferiores. Nótese que lo que respecta a díametros no hay una diferencia y lo que marca una diferencia es el número de individuos, ademas la mayoría de estas especies tenían hábitos arbustivos en las que los díametros son pequeños y los arboles que existen tambien son díametros pequeños y sus máximas alturas no pasan de $7 \mathrm{~m}$. Nótese que hay una combinación de especies de bosque montano (Escallonia myrtilloides, etc, ...) y páramo arbustivo (Macleania rupestris, etc., ...).

Encontramos las siguientes especies que tienen algún grado de amenaza: Axinaea quitensis (NT), Miconia papillosa (LC), Grosvenoria campii (EN), Verbesina latisquama (LC), Cyathea caracasana (CITES) [21].

El índice de diversidad de Simpson (Cuadro 2) indica la relación entre riqueza o número de especies y la abundancia o número de individuos por especies en cualquier sitio dado [22]. En cuanto a la relación del número de especies con individuos tenemos un índice de diversidad de Simpson de 095 que nos indica que es alto ya que se acerca a 1. En este caso ocurre porque el resultado de 33 especies y 249 individuos, son valores altos.

\section{Discusión}

Por registros de herbario de las 32 especies encontrados, 25 son de bosque montano, 9 de páramo arbustivo y solo 3 esppecies pueden estar tanto en bosque como en páramo; por esta razón decimos que es un bosque montano análago porque la mayoría de especies son de éstas y la particularidad es que su habito y altura son de menor tamaño que correspondería a un bosque montano típico, por todo ello decimos que es una 'vegetación análoga' de bosque montano. En [23] manifiesta que la vegetación tiene un proceso importante de regeneración natural, que no necesariamente es homogénea y como resultado se obtiene vegetación de bosque montano y páramo a ritmo lento. 


\section{Table 1}

Listado de especies con sus respectivos IV.

\begin{tabular}{|c|c|c|c|c|c|c|c|}
\hline Familia & Especie & Individuos & DAP & AB & DR & DMR & IV \\
\hline ESCALLONIACEAE & $\begin{array}{l}\text { Escallonia } \\
\text { myrtilloides }\end{array}$ & 20 & 400 & 125.664 & 8,03 & 8,03 & 8,03 \\
\hline ERICACEAE & Macleania rupestris & 19 & 380 & $113.411,76$ & 7,63 & 7,63 & 7,63 \\
\hline ERICACEAE & $\begin{array}{l}\text { Vaccinium } \\
\text { floribundum }\end{array}$ & 15 & 300 & 70.686 & 6,02 & 6,02 & 6,02 \\
\hline ASTERACEAE & Gynoxis buxifolia & 14 & 280 & $61.575,36$ & 5,62 & 5,62 & 5,62 \\
\hline MELASTOMATACEAE & Miconia bracteolata & 14 & 280 & $61.575,36$ & 5,62 & 5,62 & 5,62 \\
\hline ASTERACEAE & $\begin{array}{l}\text { Bacharis } \\
\text { teindalensis }\end{array}$ & 12 & 240 & $45.239,04$ & 4,82 & 4,82 & 4,82 \\
\hline BLECHNACEAE & Blechnum loxensis & 12 & 120 & $11.309,76$ & 4,82 & 4,82 & 4,82 \\
\hline CELASTRACEAE & Maytenus vertillata & 10 & 200 & 31.416 & 4,02 & 4,02 & 4,02 \\
\hline HYPERICACEAE & $\begin{array}{l}\text { Hypericum } \\
\text { laricifolium }\end{array}$ & 10 & 200 & 31416 & 4,02 & 4,02 & 4,02 \\
\hline MELASTOMATACEAE & Tibouchina lepidota & 10 & 200 & 31.416 & 4,02 & 4,02 & 4,02 \\
\hline MELASTOMATACEAE & $\begin{array}{l}\text { Brachyotum } \\
\text { ledifolium }\end{array}$ & 10 & 200 & 31.416 & 4,02 & 4,02 & 4,02 \\
\hline SYMPLOCCACEAE & $\begin{array}{l}\text { Symploccos } \\
\text { quitense }\end{array}$ & 10 & 200 & 31.416 & 4,02 & 4,02 & 4,02 \\
\hline ELAEOCARPACEAE & Vallea stipularis & 9 & 180 & $25.446,96$ & 3,61 & 3,61 & 3,61 \\
\hline ERICACEAE & $\begin{array}{l}\text { Disterigma } \\
\text { empetrifolium }\end{array}$ & 9 & 180 & $25.446,96$ & 3,61 & 3,61 & 3,61 \\
\hline MELASTOMATACEAE & Miconia crocea & 9 & 180 & $25.446,96$ & 3,61 & 3,61 & 3,61 \\
\hline MELASTOMATACEAE & Axinaea quitensis & 7 & 140 & $15.393,84$ & 2,81 & 2,81 & 2,81 \\
\hline ASTERACEAE & Grosvenoria campii & 6 & 120 & $11.309,76$ & 2,41 & 2,41 & 2,41 \\
\hline CYATHEACEAE & $\begin{array}{l}\text { Cyathea } \\
\text { caracasana }\end{array}$ & 5 & 100 & 7854 & 2,01 & 2,01 & 2,01 \\
\hline ERICACEAE & $\begin{array}{l}\text { Guaultheria } \\
\text { glomerata }\end{array}$ & 5 & 100 & 7854 & 2,01 & 2,01 & 2,01 \\
\hline GUNNERACEAE & Gunnera brephogea & 5 & 100 & 7854 & 2,01 & 2,01 & 2,01 \\
\hline MELASTOMATACEAE & Miconia papillosa & 5 & 100 & 7854 & 2,01 & 2,01 & 2,01 \\
\hline POACEAE & Neurolepis aristata & 5 & 100 & 7854 & 2,01 & 2,01 & 2,01 \\
\hline ASTERACEAE & $\begin{array}{l}\text { Diplostephium } \\
\text { hartwegii }\end{array}$ & 4 & 80 & 5026,56 & 1,61 & 1,61 & 1,61 \\
\hline CUNNONIACEAE & $\begin{array}{l}\text { Weinmania } \\
\text { mariquitae }\end{array}$ & 4 & 80 & 5026,56 & 1,61 & 1,61 & 1,61 \\
\hline MELASTOMATACEAE & $\begin{array}{l}\text { Miconia } \\
\text { corymbiformis }\end{array}$ & 4 & 80 & 5026,56 & 1,61 & 1,61 & 1,61 \\
\hline ROSACEAE & $\begin{array}{l}\text { Hesperomeles } \\
\text { obtusifolia }\end{array}$ & 4 & 80 & 5026,56 & 1,61 & 1,61 & 1,61 \\
\hline ROSACEAE & $\begin{array}{l}\text { Hesperomeles } \\
\text { ferruginea }\end{array}$ & 3 & 60 & 2827,44 & 1,2 & 1,2 & 1,2 \\
\hline BUXACEAE & $\begin{array}{l}\text { Styloceras } \\
\text { laurifolium }\end{array}$ & 2 & 40 & 1256,64 & 0,8 & 0,8 & 0,8 \\
\hline
\end{tabular}




\section{Table 1}

Continued.

Familia
POLYGALACEAE
SOLANACEAE
ASTERACEAE
ASTERACEAE
PIPERACEAE

\begin{tabular}{l|l} 
Especie & Individuos \\
$\begin{array}{l}\text { Monnina } \\
\text { phillyreoides }\end{array}$ & 2 \\
$\begin{array}{l}\text { Sessea vestita } \\
\text { Verbesina }\end{array}$ & 2 \\
latisquama & 1 \\
Barnadesia arborea & 1 \\
\hline Piper nubigenum & 1 \\
& 249
\end{tabular}

DAP
40
40
20
20
20

$\mathbf{A B}$
1256,64
1256,64
314,16
314,16
314,16
$590.424,45$

\begin{tabular}{l|l|}
\hline DR & DM \\
\hline 0,8 & 0,8 \\
\hline 0,8 & 0,8 \\
0,4 & 0,4 \\
\hline 0,4 & 0,4 \\
0,4 & 0,4 \\
\hline 100 & 100 \\
\hline
\end{tabular}

\begin{tabular}{|l|l|}
\hline DMR & IV \\
\hline 0,8 & 0,8 \\
\hline 0,8 & 0,8 \\
\hline 0,4 & 0,4 \\
\hline 0,4 & 0,4 \\
\hline 0,4 & 0,4 \\
\hline 100 & 100 \\
\hline
\end{tabular}

En otros estudios donde se han encontrado de 14 a 23 especies [24]. Gentry en sus transectos de 0.1 Ha a más de 3000 m en Ecuador encontró 40 especies en CorazónCarchi y 37 en Pasochoa, Pichincha [14]. Sin embargo, Gentry utilizó un diseño de corte transversal más disperso (dos transectos de $2 \times 50 \mathrm{~m}$ para cada transecto individual de $4 \times 50 \mathrm{~m}$ en el presente estudio), lo que explicaría al menos en parte, algunos de los mayores niveles de diversidad reportados en sus estudios. En el transecto encontré 33 especies en 0,1 Ha, no hay estudios previos en este tipo de vegetación, además observando el listado hay una mezcla de especies de bosque montano como es el caso de Escallonia myrtilloides que fue el máximo valor pero lo siguen muy de cerca Macleania rupestris entre otras que se oes puede observar en páramo arbustivo o claros de bosque. Y tal vez por eso la alta prescencia de especie ya que mas que seguro que esto sea una zona de transición entre páramo arbustivo y bosque montano.

Referencia [25] mencionan a Clethra, Clusia, Escallonia, Freziera, Gaultheria, Hedyosmum, Hesperomeles, Miconia, Morella, Oreopanax, Podocarpus, Prumnopitys, Symplocos y Weinmannia como los géneros dominantes de bosque montano neotropical entre 2.500 y $3.500 \mathrm{~m}$. En nuestro estudio tenemos 8 de 14 géneros enunciados, ya que es una zona de transición. Además incorporar a Vallea y Berberis géneros importantes en este tipo de vegetación. En cuanto a familias corroboramos también con 15, tenemos Melastomataceae: 7 especies, Asteraceae: 6 especies, Ericaceae: 4 especies; Rosaceae: 2 especies.

La explicación de tanto especies de páramo arbustivo y bosque montano alto no están tan claros, ya que fisionomicamente fue un bosque montano alto esta zona en estudio. Además no se han encontrado estudios en otras zonas de transición, pero por ello se encuentra un número alto de especies de dos tipos de vegetación.

\section{Conclusiones}

No hay una explicación clara por que hay especies de bosque montano y páramo arbustivo en el sector, porque la fisionomia es de un bosque montano alto, por lo que recomendamos hacer seguimientos a largo plazo para estudiar su dinámica. Una 
ventaja es que se tiene mas especies de bosque mntano y páramo arbustivo es decir este sector es mas diverso.

\section{References}

[1] Talbot RW, Harris RC, Browell EV, Gregory GL, Sebachery DI, Beck SM. Distribution and Geochemistry of Aerosols in the Tropical North Atlantic Troposphere: Relationship to Saharan Dust. Journal of Geophysical Research. 1986; 91:5173-5182.

[2] Brown A, Kappelle M. 2001 Introducción a los bosques nublados de Latinoamérica [Introduction to the cloudy forests of Latin America]. Una Síntesis regional. 2001; Pp. 25-40. En: Kappelle M, Brown AD (eds.) Bosques Nublados del Neotrópico. Instituto Nacional de Biodiversidad (INBio), Santo Domingo de Heredia. Spanish

[3] Araujo-Murakami A. Estructura y diversidad de plantas leñosas en un bosque amazónico preandino en el sector del Río Quendeque [Structure and diversity of woody plants in a pre-Andean Amazon forest in the sector of the Quendeque River]. Parque Nacional Madidi, Bolivia. Ecología en Bolivia: 2005; 40(3): 304-324. Spanish

[4] Whittaker RH. Communities and ecosystems. Nueva York: MacMillan Publishing; 1975. 385 p.

[5] Sierra R. Propuesta preliminar de un sistema de clasificación de vegetación para el Ecuador continental [Preliminary proposal for a vegetation classification system for continental Ecuador]. Proyecto INEFAN/GEF-BIRF y EcoCiencia. Quito, Ecuador; 1999. Spanish

[6] Pauli H, Gottfried M, Hohenwallner D, Reiter K, Casale R, Grabherr G. Manual para el trabajo de campo del proyecto GLORIA [Manual for field work of the GLORIA project,Internet]. Viena: Universidad de Viena, Instituto de ecología y conservación biológica; 2003 [Consultado julio 10 del 2019]. Disponible en: http://www.gloria.ac.at/downloads/GLORIA_MS4_Web_espanol.pdf. Spanish

[7] Azócara A. (1981). La flora de los páramos [The flora of the paramos,Internet]. Universidad de los Andes: Venezuela; 1981. Disponible en: http://www.ciens.ula.ve/icae/publicaciones/paramo/articulos_ por_autorb.php. Spanish

[8] Josse C, Mena P, Medina G. La Biodiversidad de los páramos [Biodiversity in the paramos, Internet]. Serie Páramo GTP: 2000. Disponible en: http://paramosecuador.org.ec. Spanish

[9] Mena P, Hofstede R. Los páramos ecuatorianos [The Ecuatorian paramos]. Botánica Económica de los Andes Centrales. 2006: 91-109. Spanish

[10] León-Yánez S. La flora de los páramos ecuatorianos [The flora in Ecuatorian paramos]. La biodiversidad de los páramos. Quito: Serie Páramo. 2000;7: 5-21. Spanish

[11] Sklenář P, Luteyn JL, Ulloa C, Jørgensen PM, y Dillon MO. Flora Genérica de los Páramos: Guía llustrada de las Plantas Vasculares [Generic Flora of the Páramos: Illustrated Guide of Vascular Plants]. Memoirs of the New York Botanical Garden: 2005;92: 3-499. Spanish

[12] Hofstede R, Calles J, López V et al. Los Páramos Andinos ¿Qué sabemos? Estado de conocimiento sobre el impacto del cambio climático en el ecosistema páramo [The Andean Páramos, What do we know? State of knowledge on the impact of climate change on the páramo ecosystem]. Quito, Ecuador: UICN; 2014. Spanish

[13] León-Yánez S. Los Páramos Andinos ¿Qué sabemos? Estado de conocimiento sobre el impacto del cambio climático en el ecosistema páramo [The Andean Páramos, What do we know? State of knowledge on the impact of climate change on the páramo ecosystem]. Quito, Ecuador: UICN; 2014. Spanish

[14] Phillips O, Miller JS. Global patterns of plant diversity: Alwyn H. Gentry's forest transect data set. Monographs in Systematic Botany from the Missouri Botanical Garden. 2002; 89: 1-319.

[15] Ceron C. Manual de botánica, sistemática, etnobotánica y métodos de estudio en el Ecuador [Manual of botany, systematics, ethnobotany and study methods in Ecuador]. Ecuador: Herbario "Alfredo Paredes" QAP, Escuela de Biología de la Universidad Central del Ecuador. 2003. Spanish

[16] Caranqui J, Romero F. (2014, May). Diversidad y similitud arborea de los bosques montanos de la provincia de Chimborazo [Diversity and arboreal similarity of the montane forests of the province of Chimborazo]. In XI Congreso deficiencia y Tecnología. Ecuador: ESPE; 2014 May. p 11-17. Spanish

[17] Caranqui J. Estudios básicos de bosques montanos en el centro del Ecuador [Basic studies of montane forests in central Ecuador]. Alemania: Editorial Académica Española; 2011. p 67. Spanish

[18] Jørgensen PM, León-Yánez S. Catalogue of the Vascular Plants of Ecuador. Missouri Botanical Garden. 1999.

[19] Trópicos base de datos [Internet]. Missouri Botanical Garden. [Consulta de internet 2019 Ago 31] Disponible en: http://www.tropicos.org 
[20] Bray JR, Curtis JT. An ordination of the upland forest communities of southern Wisconsin. Ecological Monographs. 1957; 27(4): 325-349

[21] Valencia R. Libro rojo de las plantas endémicas del Ecuador []. Ecuador: Herbario QCA, Pontificia Universidad Católica del Ecuador. 2000.

[22] Smith L, Smith T. Ecología [Ecology]. Madrid: Pearson Education; 2001. p 664. Spanish

[23] Caranqui J, Suarez D. (2016). Análisis de la regeneración natural después de la explotación de pino en el páramo de Tamboloma [Analysis of the natural regeneration after the exploitation of pine in the paramo of Tamboloma,Internet] Tungurahua-Ecuador; 2016. Disponible en: http://dspace.espoch.edu. ec/handle/123456789/4623

[24] Gentry AH. Patterns of diversity and floristic composition in neotropical montane forest. 1995. p103-126. In: Churchill S P, Balslev H, Forero E, Luteyn JL. Biodiversity and conservation of neotropicals montane forests. The New York Botanical Garden, Bronx.

[25] Kessler M, Beck SG. En: Kappelle M, Brown AD. Bosques nublados del Neotrópico [Cloudy forests of the Neotropics,eds]. Bolivia: Instituto Nacional de Biodiversidad, InBio, Santo Domingo de Heredia; 2001. p. 581-622. 